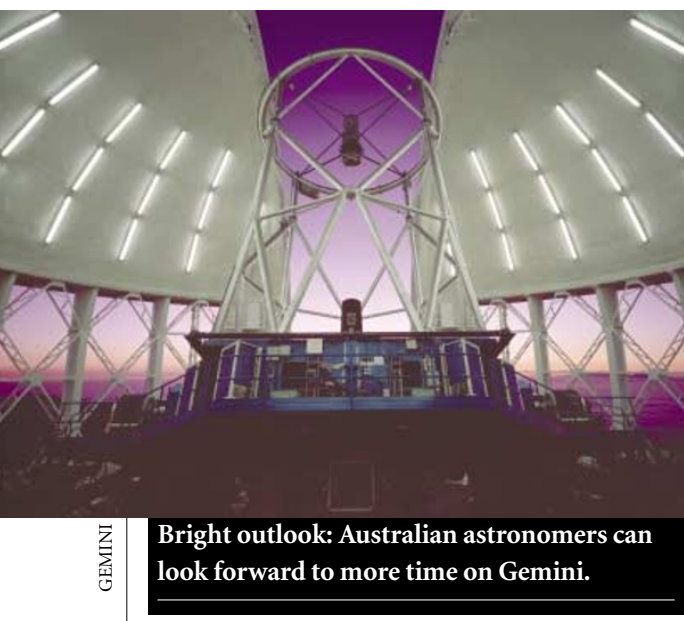

Funding bonanza for astronomy and biotech in Australia

Peter Pockley, Sydney

In a bid to answer perennial charges that it has short-changed science, the Australian government is to provide $\mathbf{A} \$ 151$ million (US\$80 million) over five years for 15 major facility projects.

Astronomers won the largest single grant, gaining A \$23.5 million for studies using the international Gemini telescopes in Chile and Hawaii. But biotechnology was the most favoured field overall, with with total funds of $\mathbf{A} \mathbf{4 7}$ million for four projects, reflecting the government's emphasis on research with likely commercial applications.

Intense competition for the grants, which were announced on 21 August, meant that only about one in six of the 86 applicant projects was successful.

Nonetheless, Australian astronomers can now double their observing time on the 8-metre Gemini telescopes. They will also develop instruments to help Australia's bid to host the proposed international Square Kilometre Array radiotelescope.

The second-largest single grant, of A \$18 million, will help to establish a neuroscience facility based primarily in Melbourne for studying and treating schizophrenia and Alzheimer's and Parkinson's diseases. Stem-cell research got a smaller grant for a new cell-engineering centre at Monash University in Melbourne.

Research at synchrotron light sources will continue with a A $\$ 14.8$-million grant for Australian beamlines at facilities in Japan and the United States.

Other projects to win backing include facilities for proteomics and genomics, a photonics foundry, a livestock genetic database, a marine station for oil and gas exploration in tropical waters, and a centre for wine research.

\title{
Canada unveils plans to build nanotechnology centre...
}

\section{David Spurgeon, Montreal}

Canada is set to invest Can $\$ 120$ million (US\$78 million) over five years in nanotechnology, creating a new institute based in the western province of Alberta.

On 17 August, Prime Minister Jean Chretien and Ralph Klein, premier of Alberta, unveiled plans for the Institute for Nanotechnology, which will be attached to the University of Alberta in Edmonton. Employing about 200 people, the institute will be funded jointly by the federal and provincial governments.

Federal support for the institute will come from Canada's National Research Council (NRC). It will be the first NRC institute to be governed jointly with the host university. The centre will collaborate closely with the University of Alberta, the NRC says, making joint appointments and sharing research facilities.

Peter Hackett, the NRC's vice-president for research, says he is "overjoyed" by the announcement. "Canada has decided to make a major investment in an emerging field. We did this before as a nation in 1984 in biotechnology - with the Biotechnology Research Institute in Montreal - and that's turned out to be very successful."

Hackett says that the new institute will be "interdisciplinary from day one". The institute will offer "a different culture and a different way of looking at engineering, chemistry,

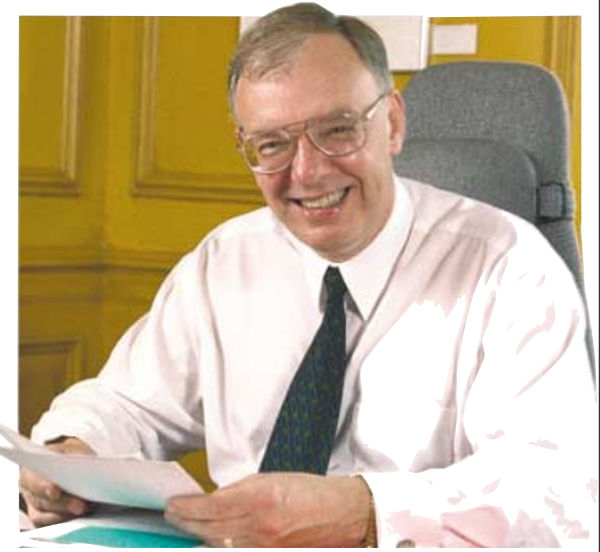

Thinking small: Peter Hackett says the nanotech institute will improve scientific cooperation.

physics and biology", he says. "Everyone will share this perspective of the nano-world."

Michael Brett, a researcher in nanostructure and thin-film engineering at the University of Alberta, agrees. The cooperative arrangement between the university and the NRC will create "a much richer institute", he says, because of the mix of scientists and graduate students and the availability of university facilities.

The main areas of investigation at the institute will relate to biomaterials, biomedical devices, computing and quantum computing, and catalysts.

| http://www.nrc.ca/nanotech

\section{. . . as mathematicians beat retreat to Alberta}

\section{Erica Klarreich}

North American mathematicians will soon be able to immerse themselves in their thoughts at a purpose-built hideaway high in the Canadian Rockies.

When it opens in 2003, the Banff International Research Station will allow invitees to "live, eat and breathe mathematics", says Nassif Ghoussoub, director of the Pacific Institute for the Mathematical Sciences (PIMS) in Vancouver.

Inspired by existing retreats for mathematicians in Oberwolfach, Germany, and Luminy, France, the research station will invite up to 40 mathematicians at a time to participate in intense, five-day workshops.
"We've seen from Oberwolfach and Luminy that this kind of setting is a fantastically successful way to advance a mathematics research agenda," says Philippe Tondeur, director of the mathematics division at the US National Science Foundation (NSF).

The station will cost about $\$ 1.5$ million per year to run, and will be supported in roughly equal parts by the NSF, the Natural Sciences and Engineering Research Council of Canada, the Alberta Science and Research Authority and PIMS. The NSF and PIMS have already announced their support, and the other partners are expected to confirm their participation on 24 September.

As many as 1,600 mathematicians could pass through Banff each year. In addition to the five-day workshops, it will accommodate groups of 10-15 mathematicians to work for up to four weeks on specific research topics.

http://www.pims.math.ca/birs

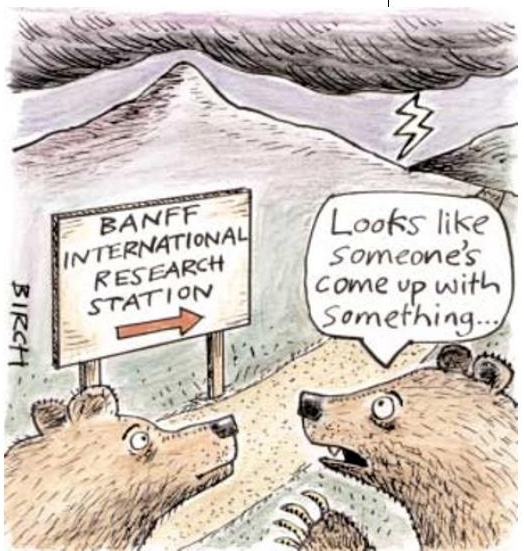

